saccharide, but later related the activity to virus-like particles present in the fungus, and ultimately found that the interferon inducer in this anti-viral preparation was viral RNA. Almost simultaneously, E. B. Chain's group at Imperial College, London, in collaboration with M. Hollings et al. at the Glasshouse Crops Research Institute, Littlehampton, published a definitive report that the active material is a double stranded mycophage RNA (Nature, 218, 542; 1968).

Taking this lead, several groups have investigated the therapeutic properties of other double stranded viral RNAs and also synthetic RNAs. These substances have great potentialities-probably even more than the few known double stranded RNA viruses might offer-if only the toxic properties are not a handicap. Toxicity is still a serious problem. This issue of Nature contains three reports documenting the side effects of poly-I.poly-C. Absher and Stinebring (page 715) present evidence that it is similar to endotoxin in its capacity to stimulate interferon-and in its toxicity. Lindsay et al. (page 717), by studying the pyrogenicity of poly-I.poly-C, seek evidence which will help to decide whether these inducers exert their effects by inducing synthesis de novo or by causing the release of preformed interferon. At present, opinion is divided on this point; it is quite likely that both induction and release occur. The third article (page 718), by Adamson and Fabro, concerns the embryotoxicity of poly-I. poly-C. The action of interferon stimulators in embryo tissue is of particular relevance to the possible uses for such substances in tumour therapy-both tumours and embryos represent rapidly growing tissues. H. B. Levy et al., and also L. D. Zeleznick and B. K. Bhuyan, have shown that poly-I.poly-C is effective against tumours in mice and rats and this property is apparently not restricted to viral tumours (here it is tempting simply to attribute it to interferon stimulation) but also in non-viral tumours. I. Gresser's group in Paris have found that interferon itself is potent against both types of tumour.

An air of uncertainty-and at the same time great activity-seems to pervade the interferon field at present. On the one hand, there is the prospect of potential therapeutic agents like poly-I.poly-C; on the other hand, there is a recent swing in some quarters back to interferon itself, even if it is elusive and hard to purify.

\section{ELECTRONS IN SOLIDS}

\section{Photoelectrons are Useful}

\section{from our Solid State Physics Correspondent}

THE behaviour of inter-particle forces in a solid can often be revealed by tracking some mobile particle, for example, an X-ray or ultraviolet photon, the motion of which will be affected by interactions within the solid. A different technique, gaining extra applications each year, is based on the analysis of the electrons emitted from the solid after the absorption of a photon from an external beam. This photoelectron method has already been seized on by chemists as a means of studying molecular orbitals and other properties not readily accessible by conventional spectroscopy.

Two recent articles in Physical Review Letters show that measurements of the spin polarization of photoelectrons can give useful information about the electron states in a metal. R. E. De Wames and L. A. Vredevoe (Phys. Rev. Lett., 23, 123; 1969) have shown that in gadoliniumthe most suitably ferromagnetic metal for photoelectron studies - the orientation of the magnetic moments of the electrons emitted is related to the polarizations of the electrons in the conduction band by means of an electron-magnon interaction which occurs as the electron travels to the surface of the solid. At high energies, however, the $1 / E^{2}$ term in the inelastic electron-magnon cross-section guarantees that the scattering will be small, so that it should be possible to measure the polarization of the conduction band directly from the photoelectrons in this region.

There are essentially three steps in a photoelectron emission process. A conduction electron is excited to a high energy state by absorbing a photon and then drifts to the surface, where it may or may not be emitted. In this procedure, the spin polarization may be affected by electron-magnon scattering or a spin deperdent surface-barrier potential. De Wames and Vredevoe show that even on a simple Heisenberg model, the electron-magnon collisions have a decisive effect on the electron spins. This is contrary to the assumption of Busch et al. (Phys. Rev. Lett., 22, 597; 1969), who carried out measurements on gadolinium at low energies and interpreted their measured polarization of +5 per cent as reflecting directly that of the conduction band. When De Wames and Vredevoe included the effect of the electron-magnon interaction, however, they found that the measured polarization would result from a conduction band polarization of around -15 per cent. They point out that to achieve a direct correlation requires considerably higher energies than those used by Busch et al.

A novel use for photoelectrons has also been put forward by U. Fano (Phys. Rev., 178, 131; 1969), who predicts that the ionization of caesium atoms by circularly polarized light would produce spin polarized electrons. This prediction rests on the existence of a spin-orbit interaction in the continuum state of the photoelectron before it becomes free. M. S. Lubell and W. Raith (Phys. Rev. Lett., 23, 211; 1969) have tested Fano's theory by measuring the counting rate of ions produced in the photoionization of polarized caesium atoms. This quantity is directly related to the polarization of photoelectrons in the conventional experiment, but is claimed to give a better statistical accuracy. Lubell and Raith have found that Fano's assumption that there should be a non-zero cross-section arising from a spin-orbit interaction is upheld remarkably well. They made measurements for various energies and obtained a polarization curve which they interpret as firmly supporting the existence of spin-orbit coupling in the continuum state.

\section{CARTOGRAPHY}

\section{Space Age Road Map}

\section{from our Geomagnetism Correspondent}

The United States Geological Survey and the National Aeronautics and Space Administration have jointly 